\title{
Branching out: mapping the spatial expansion of the lessepsian invader mytilid Brachidontes pharaonis around the Maltese Islands
}

\author{
DAVID P. CILIA ${ }^{1}$ AND ALAN DEIDUN ${ }^{2}$ \\ ${ }^{1}$ St Paul's Missionary College, Emanuele Vitale Street, Rabat, RBT 2020, Malta, ${ }^{2}$ International Ocean Institute-Malta Operational \\ Centre, University of Malta, Msida, MSD 2080, Malta
}

\begin{abstract}
One of the several lessepsian species colonizing areas of the Mediterranean is Brachidontes pharaonis, a bivalve with the ability to form dense mytilid mats over a range of different mediolittoral substrata. Since its initial observation from the Maltese Islands in the early 1970s, the species has consolidated its presence all over the archipelago. Close examination of the entire length of the Maltese shoreline was conducted to collect quantitative and qualitative data on the mytilid and on dominant accompanying macrofaunal and macrofloral species, in what represents the first comprehensive mapping of an allochthonous species within an island territory. Brachidontes pharaonis was found to have colonized most of the northern and eastern coastal stretches of the island of Malta, preferring limestone substrata in inlets with limited wave exposure and affected by high marine concentrations of hydrocarbons and other pollutants, where it reached individual abundances exceeding 1000 individuals per square metre.
\end{abstract}

Keywords: mapping, spatial expansion, lessepsian invader mytilid, Brachidontes pharaonis, Malta

Submitted 16 December 2011; accepted 10 January 2012

\section{INTRDDUCTION}

The invasion of Red Sea species into the eastern basin of the Mediterranean through the Suez Canal is known as 'lessepsian migration' (Por, 1978). According to Zenetos et al. (2010), who give an updated list of alien marine species recorded to date from the Mediterranean Sea, a total of 955 alien marine species have been recorded from the Mediterranean, the vast majority of which (718) having being introduced in the Eastern Mediterranean and are thus, presumably, of lessepsian origin.

The taxonomy of the species discussed in this paper is in a state of flux, as until more genetic studies become available it is difficult to group or split the several Brachidontes morphological variants into one or more valid taxa.

On one hand, several geographical forms including Brachidontes pharaonis (Fischer, 1870) are considered by some authors (Lamy, 1936; Huber, 2010) to be variants of Brachidontes ustulatus (Lamarck, 1819). This is an Indo-Pacific mytilid with a highly variable shell first described as Mytilus ustulatus, with Lamarck's locus typicus for the type series being Brazil. Lamarck's description was based on specimens collected by the French explorer Nicolas Baudin, presumably during his voyage to Australia spanning from 1800 to 1803 . For this reason, Lamy (1936) argues that the locus

Corresponding author:

A. Deidun

Email: alandeidun@gmail.com typicus is incorrect and the type series is actually from the Indo-Pacific area. According to Huber (2010), the variability of this species has led to the propagation of several synonyms such as Brachidontes semistriatus Krauss, 1848, Mytilus variabilis Krauss, 1848, (and hence Brachidontes variabilis), Mytilus pharaonis Fischer, 1870 and Mytilus arabicus Jousseaume in Lamy (1919). Lamy reassessed his 1919 opinion in 1936, synonymizing $M$. arabicus, $M$. pharaonis, and $M$. variabilis with their senior synonym B. ustulatus. Huber (2010) reiterates Lamy's view and groups several forms into one vast 'ustulatuscomplex' spreading from the Red Sea (and the westward invasion, cf. Sarà et al., 2000) to Japan, individuals of which complex show a high degree of morphological plasticity.

On the other hand, molecular studies like that of Sirna Terranova et al. (2007) discover genetic divergence between separate geographical populations that are 'substantially higher' than those between species of other congeners of other mytilid genera such as Mytilus Linné, 1758. According to Sirna Terranova et al. (2007), B. variabilis actually consists of three species-B. pharaonis 'sensu lato' (to which the Red Sea and Mediterranean populations belong), an Indian Ocean B. variabilis and a Pacific Ocean B. 'variabilis'.

In this paper, the authors adhere to the name Brachidontes pharaonis (Fischer, 1870) for the sake of geographical specificity and clarity.

One of the earliest recorded and most successful lessepsian migrants in the Mediterranean Sea is indeed B. pharaonis (cf. Zenetos et al., 2010), having established large and stable populations within the basin. The species hails from the Indo-Pacific, and by 1976 it spread as far west as the Red 
Sea forming mytilid clusters (Gilboa, 1976; Safriel et al., 1980). The bivalve was first recorded in the Mediterranean Sea at Port Said just seven years after the opening of the Suez Canal in 1869, and has to date spread from the Israeli coastline, where a study covering the extent of colonization was carried out by Rilov et al. (2004), to the western coast of Sicily (Sarà et al., 2008), establishing stable populations even in the north Adriatic (De Min \& Vio, 1997). Records from the Aegean Sea are sporadic (e.g. Tenekides, 1989; Dogan et al., 2007). Sarà et al. (2000) have predicted that the species will continue its westward spread from Sicily to North Africa and Gibraltar.

The first publications mentioning Brachidontes pharaonis for the Maltese Islands (as Brachidontes variabilis (Krauss)) were those by Cachia (1975) and Lanfranco (1975); however, records of the species from October 1970, in Qawra and Baћar ić-Cagћaq, exist, together with 1973 records from St Julian's, Kalkara, St Thomas' Bay and Birżebbuga Bay (C. Cachia, personal communication). Most of these rare finds consisted of disarticulated valves, others of which occurred in 1985 in Marsaskala Bay (C. Mifsud, personal communication) (see Table 1 and Figure 1 for historical and unpublished records). Subsequent checklists (Cachia et al., 1993; Cachia, 1999; Cachia et al., 2004; Sciberras \& Schembri, 2007) mention the species with the taxon now amended to Brachidontes pharaonis (P. Fischer, 1870). In Cachia et al. (2004) it is noted that the species occurs as 'few individuals living at the lower mediolittoral, often embedded in moss-like algae'. Mifsud \& Cilia (2009) reported the first significant colonies with a mytilid cluster habitus from Birżebbuga.

Brachidontes pharaonis displays several features that are typical of invading species (Sarà et al., 2003), such as high fecundity and tolerance to variable environmental conditions, including high levels of pollution, according to Morton (1988) and Shefer (2003). The proliferation of B. pharaonis within the Mediterranean Sea, the ease with which it can be sampled and the evident displacement of indigenous species of Mytilaster Monterosato, 1883 it causes (Felsenburg \& Safriel, 1974; Safriel \& Sasson-Frostig, 1988; Rilov et al., 2002) have fuelled physiological (e. g. Sarà, 2006) and even genetic (Shefer et al., 2004; Sirna Terranova et al., 2006, 2007) studies on the species. The latter investigations have disclosed a lack of genetic structure within the Mediterranean populations for the species, suggesting a relative lack of genetic differentiation between such populations.

The mapping of a species' distribution and the documentation of shifts in such an occurrence have fascinated biologists for decades, ever more so with the ascent of invasion biology. This study represents one of the first comprehensive attempts at mapping the distribution of a non-indigenous species (B. pharaonis) conducted over the entire extent of a national territory-that of the Maltese Islands-with one other similar study being that of Rilov et al. (2004), conducted in Israel. Supplementary information on the density of the mytilid and on the ecological and physical characteristics of each of the sampling sites is also compiled.

\section{MATERIALS AND METHDDS}

A series of observations on local shores was carried out between 2009 and 2011 by the authors. A stretch of coastline at a time was explored and the presence or absence of $B$. pharaonis was noted. The entire extent of the low-lying coastline of the Maltese archipelago was investigated in this way, with only coastal stretches having a significant slope value ( $>10$ degrees; e.g. the cliffs along the western coastline of Malta and Gozo) not being sampled.

In the case of positive results, a measurement of the population density was carried out using three randomlypositioned $0.01 \mathrm{~m}^{2}$ replicates, about $1 \mathrm{~m}$ above mean sea level (as demarcated by Cystoseira spp. populations), with subsequent extrapolation to a value for one square metre. Locations with very small, scattered numbers of specimens were listed as containing 'isolated individuals'. The mytilids were not scraped off the rocks prior to counting, therefore the densities observed and reported in this study are an underestimate, consisting exclusively of superficially visible adults. Using on-site observations and maps (Government of Malta, 1993; Google Maps, 2011) the type of substratum colonized by $B$. pharaonis was recorded and coordinates of each sampling location were extracted. Predominant supralittoral to sublittoral macrofloral and macrofaunal species, in close proximity to the $B$. pharaonis populations or specimens, were identified, at least to generic level.

\section{RESULTS}

Figure 1 gives the current distribution of $B$. pharaonis in the Maltese Islands as reported by the present study. Table 1 lists all the previous records of $B$. pharaonis from the Maltese Islands, whilst Table 2 lists the findings at each individual sampling site adopted in the present study.

Table 1. Historical and unpublished records of Brachidontes pharaonis (Fischer, 1870) in the Maltese Islands.

\begin{tabular}{|c|c|c|c|c|}
\hline Date of record & Location & Number on map & Comments & Communication \\
\hline \multirow[t]{2}{*}{ X.1970 } & Qawra & 1 & NA & Charles Cachia \\
\hline & Baћar ić-Ċaghaq & 2 & & \\
\hline \multirow[t]{4}{*}{1973} & St Julian's & 3 & & \\
\hline & Kalkara Creek & 5 & & \\
\hline & St Thomas' Bay & 7 & & \\
\hline & Birżebbuga Bay & 9 & & \\
\hline VIII.1990 & Miger Ilma, below Dingli Cliffs & 10 & Live attached to rocks at waterline & Constantine Mifsud \\
\hline IX.1985 & Marsaskala Bay & 6 & In detritus from $-4 \mathrm{~m}$ & \\
\hline IX.2004 & Bahar ić-Ċaghaq & 2 & Juveniles from tidepool & \\
\hline 24.VI.2007 & Qajjenza, Marsaxlokk Bay & 8 & On fish farm nets & \\
\hline III.2010 & Rinella Bay & 4 & On old tyre near shore & \\
\hline
\end{tabular}




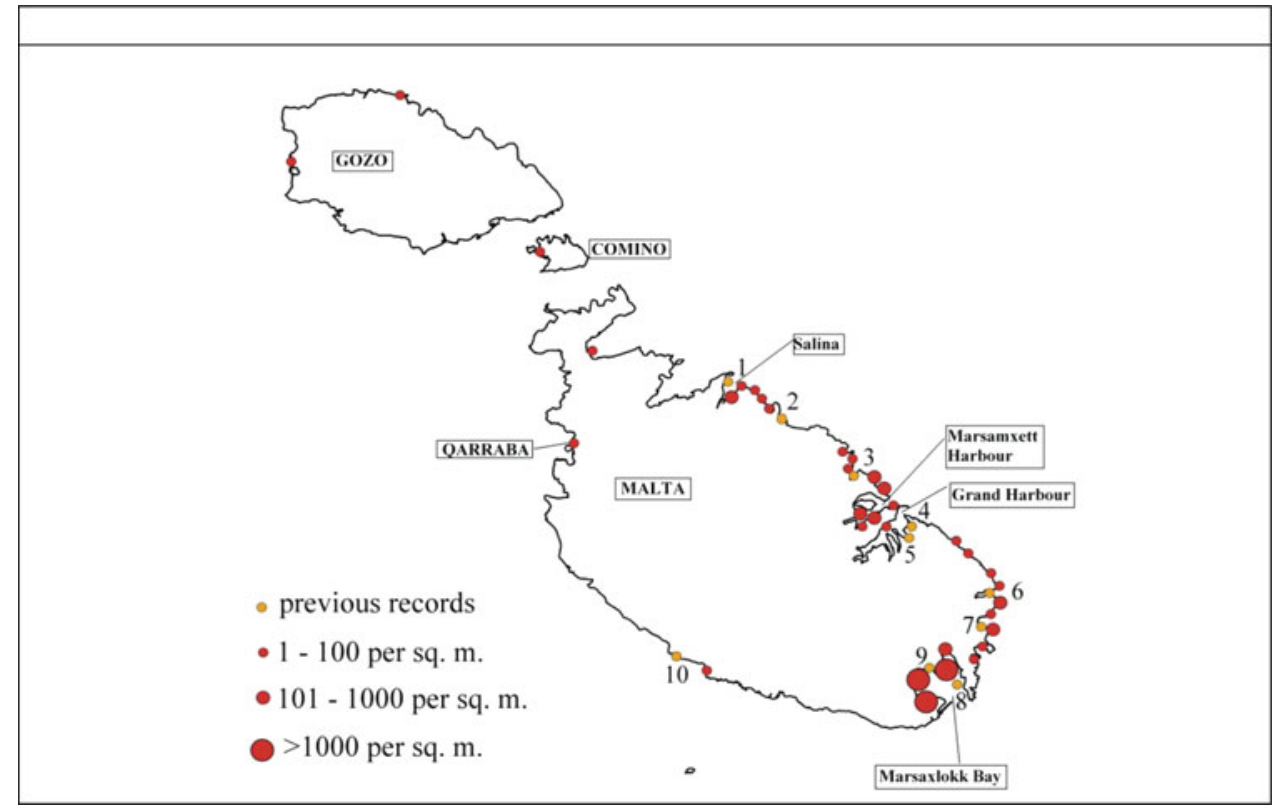

Fig. 1. Previous and current records, with the latter including population densities, for Brachidontes pharaonis (Fischer, 1870) around the Maltese Islands. Named coastal zones indicate embayments where the highest densities of $B$. pharaonis were recorded or where interesting observations about the species were made. Refer to Table 1 for the numbered records.

\section{DISCUSSIDN}

Findings from the present study seem to suggest that B. pharaonis has a predilection for sedimentary substrates (limestone), in particular Globigerina Limestone, on which it reaches the highest densities. In fact, the species occurs only as small clusters or as isolated individuals on other types of limestone, such as Upper Coralline or Lower Coralline Limestone. Besides limestone substrates, $B$. pharaonis exhibited a high degree of environmental plasticity in that it was also recorded in the present survey from concrete, wood, a discarded seaborne tyre and even from within vermetid (Dendropoma petraeum (Monterosato, 1884) and Vermetus sp.) reefs. In the westernmost record for the island of Malta, within the Qarraba area (Figure 1), the species was even observed inhabiting empty boreholes of Lithophaga lithophaga (Linné, 1758).

Although surveys conducted by malacologists since 1970 in the Maltese Islands were not systematic ones but just snapshot studies restricted to point locations only, such that $B$. pharaonis might have been under-reported in the past, findings of the present study suggest that this species has greatly expanded its range in the archipelago in recent years, reaching, in some areas, the same densities reported, for example, from a hyperhaline saltpan in western Sicily (Sarà, 2006; Sarà et al., 2008).

The first record of $B$. pharaonis in the Maltese Islands was at Qawra (point 1 in Figure 1), made in 1970-since then, the species has expanded its range within the archipelago, being recorded also in small densities from the islands of Comino and Gozo (Figure 1). On these two islands, B. pharaonis is restricted mainly to concrete jetties, suggesting that vessels are acting as vectors, at least in initial stages, in proliferating the species.

Displacement of the native Mediterranean mytilid Mytilaster minimus (Poli, 1795) is an often-cited effect of invasion of B. pharaonis (e.g. Safriel \& Sasson-Frostig, 1988; Rilov et al., 2004). In many of the sampled localities, an absence of $M$. minimus was noted; in a small number of localities, like Baћar ić-Caghaq, Balluta and some areas in Valletta (Table 2), a degree of sympatry betwen the two species was observed; however, the density of $M$. minimus was always much lower than that of the invasive species and on no occasion was a dense cluster of M. minimus observed. It is impossible to accurately define the extent of displacement of $B$. pharaonis over M. minimus simply because there are no previous studies on the latter's population densities on the Maltese coastline. However, shell sizes exceeding the $10 \mathrm{~mm}$ normally attained by $M$. minimus were very rarely observed in areas where both species were present. Safriel \& Sasson-Frostig (1988) hypothesize that the two species are able to coexist due to different environmental preferences and an extinction-immigration continuum. Juvenile specimens of another mytilid species, Mytilus galloprovincialis Lamarck, 1819, were encountered within the St Thomas' Bay community.

In a few places, like Spinola Bay, Manoel Island and the Msida marina (Table 1), high populations of Ostreola stentina (Payraudeau, 1826) seem to inhibit clustering of B. pharaonis, creating 'breaks' in what would otherwise be a complete stretch of Brachidontes-colonized coast. A wooden boat observed at the Spinola inlet yielded a vast population of $O$. stentina yet only a single, mature, individual of $B$. pharaonis. Two main reasons are postulated for this competitive exclusion-the size and complex nature of $O$. stentina clusters make it more difficult for larva of $B$. pharaonis to settle on the substratum, while their larger and more extensive filterfeeding apparatus may competitively inhibit the invasive species from obtaining the required nutrition. In addition, $O$. stentina is seemingly less selective as regards substratum (colonizing concrete walls more frequently) and is probably much more resistant to polluted water, such as in the innermost parts of the Msida marina, where it reaches very high population densities. In Sliema, areas containing juveniles of another lessepsian migrant bivalve Pinctada radiata (Leach, 1814) lacked any specimens of the mytilid. 
Table 2. Populations of Brachidontes pharaonis (Fischer, 1870) in the Maltese Islands as discovered during the present research. Records are sorted clockwise beginning from Dwejra, Gozo.

\begin{tabular}{|c|c|c|c|c|}
\hline Date of record & Location & Coordinates & Density & Substrate \\
\hline 09.VI.2011 & Dwejra, Gozo & $36^{\circ} 03^{\prime} 13^{\prime \prime} \mathrm{N} 14^{\circ} 11^{\prime} 29^{\prime \prime} \mathrm{E}$ & Isolated individuals & $\begin{array}{l}\text { Weathered/eroded concrete } \\
\text { platform }\end{array}$ \\
\hline 09.VI.2011 & $\begin{array}{l}\text { Qbajjar Bay, Marsalforn, } \\
\text { Gozo }\end{array}$ & $36^{\circ} 0^{\prime} 37^{\prime \prime} \mathrm{N} 14^{\circ} 15^{\prime} 07^{\prime \prime} \mathrm{E}$ & $\sim_{10 \mathrm{~m}^{-2}}$ & $\begin{array}{l}\text { Lower and Middle } \\
\text { Globigerina Limestone }\end{array}$ \\
\hline 13.VI.2011 & Blue Lagoon, Comino & $36^{\circ} \mathrm{Oo}^{\prime} 51^{\prime \prime} \mathrm{N} 14^{\circ} 19^{\prime} 28^{\prime \prime} \mathrm{E}$ & Isolated individuals & $\begin{array}{l}\text { Upper Globigerina Limestone } \\
\text { coastline }\end{array}$ \\
\hline 18.V.2011 & Ghadira, Mellieћa & $35^{\circ} 58^{\prime} 07^{\prime \prime} \mathrm{N} 14^{\circ} 21^{\prime} 08^{\prime \prime} \mathrm{E}$ & Isolated individuals & Upper Coralline Limestone \\
\hline \multirow[t]{6}{*}{ 06.VI.2011 } & $\begin{array}{l}\text { Baћar ić-Ċagћaq-Salini } \\
\text { stretch }\end{array}$ & $35^{\circ} 57^{\prime} 14^{\prime \prime} \mathrm{N} 14^{\circ} 26^{\prime} 16^{\prime \prime} \mathrm{E}$ & None & $\begin{array}{l}\text { Lower Coralline Limestone } \\
\text { coastline }\end{array}$ \\
\hline & 06.VI.2011 & $35^{\circ} 57^{\prime} 06^{\prime \prime} \mathrm{N} 14^{\circ} 26^{\prime} 37^{\prime \prime} \mathrm{E}$ & None & \\
\hline & 06.VI.2011 & $35^{\circ} 56^{\prime} 52^{\prime \prime} \mathrm{N} 14^{\circ} 26^{\prime} 47^{\prime \prime} \mathrm{E}$ & Isolated individuals & \\
\hline & 06.VI.2011 & $35^{\circ} 56^{\prime} 41^{\prime \prime} \mathrm{N} 14^{\circ} 26^{\prime} 57^{\prime \prime} \mathrm{E}$ & $\sim_{10} \mathrm{~m}^{-2}$ & \\
\hline & 06.VI.2011 & $35^{\circ} 56^{\prime} 40^{\prime \prime} \mathrm{N} 14^{\circ} 27^{\prime} 09^{\prime \prime} \mathrm{E}$ & Isolated individuals & \\
\hline & 06.VI.2011 & $35^{\circ} 56^{\prime} 37^{\prime \prime} \mathrm{N} 14^{\circ} 27^{\prime} 19^{\prime \prime} \mathrm{E}$ & None & \\
\hline 06.VI.2011 & $35^{\circ} 56^{\prime} 25^{\prime \prime} \mathrm{N} 14^{\circ} 27^{\prime} 25^{\prime \prime} \mathrm{E}$ & $\sim_{100 \mathrm{~m}^{-2}}$ & $\begin{array}{l}\text { Upper Globigerina } \\
\text { Limestone coastline }\end{array}$ & \\
\hline 05.XII.2010 & Pembroke & $35^{\circ} 56^{\prime} 14^{\prime \prime} \mathrm{N} 14^{\circ} 28^{\prime} 24^{\prime \prime} \mathrm{E}$ & None & $\begin{array}{l}\text { Lower Coralline Limestone } \\
\text { coastline }\end{array}$ \\
\hline 05.XII.2010 & & $35^{\circ} 56^{\prime} 13^{\prime \prime} \mathrm{N} 14^{\circ} 28^{\prime} 40^{\prime \prime} \mathrm{E}$ & None & \\
\hline 05.XII.2010 & & $35^{\circ} 56^{\prime} 10^{\prime \prime} \mathrm{N} 14^{\circ} 28^{\prime} 53^{\prime \prime} \mathrm{E}$ & None & $\begin{array}{l}\text { Vermetid reef on Lower } \\
\text { Coralline Limestone }\end{array}$ \\
\hline 05.XII.2010 & & $35^{\circ} 55^{\prime} 53^{\prime \prime} \mathrm{N} 14^{\circ} 29^{\prime} 14^{\prime \prime} \mathrm{E}$ & None & $\begin{array}{l}\text { Lower Coralline Limestone } \\
\text { coastline }\end{array}$ \\
\hline 30.VI.2011 & $\begin{array}{l}\text { St George's Bay area, St } \\
\text { Julian's }\end{array}$ & $35^{\circ} 55^{\prime} 35^{\prime \prime} \mathrm{N} 14^{\circ} 29^{\prime} 16^{\prime \prime} \mathrm{E}$ & Isolated individuals & $\begin{array}{l}\text { Artificially modified Lower } \\
\text { Coralline Limestone } \\
\text { coastline }\end{array}$ \\
\hline 30.VI.2011 & & $35^{\circ} 55^{\prime} 34^{\prime \prime} \mathrm{N} 14^{\circ} 29^{\prime} 25^{\prime \prime} \mathrm{E}$ & Isolated individuals & $\begin{array}{l}\text { Lower Coralline Limestone } \\
\text { coastline with concrete } \\
\text { patches }\end{array}$ \\
\hline 30.VI.2011 & & $35^{\circ} 55^{\prime} 32^{\prime \prime} \mathrm{N} 14^{\circ} 29^{\prime} 16^{\prime \prime} \mathrm{E}$ & None & Sand \\
\hline 30.VI.2011 & Spinola Bay area, Sliema & $35^{\circ} 55^{\prime} 25^{\prime \prime} \mathrm{N} 14^{\circ} 29^{\prime} 37^{\prime \prime} \mathrm{E}$ & Isolated individuals & $\begin{array}{l}\text { Lower Coralline Limestone } \\
\text { coastline }\end{array}$ \\
\hline 30.VI.2011 & & $35^{\circ} 55^{\prime} 22^{\prime \prime} \mathrm{N} 14^{\circ} 29^{\prime} 44^{\prime \prime} \mathrm{E}$ & Isolated individuals & \\
\hline 30.VI.2011 & & $35^{\circ} 55^{\prime} 13^{\prime \prime} \mathrm{N} 14^{\circ} 29^{\prime} 43^{\prime \prime} \mathrm{E}$ & Isolated individuals & \\
\hline 21.IX.2010 & & $35^{\circ} 55^{\prime} 10^{\prime \prime} \mathrm{N} 14^{\circ} 29^{\prime} 25^{\prime \prime} \mathrm{E}$ & $<_{10} \mathrm{~m}^{-2}$ & Wooden fishing boat \\
\hline 21.IX.2010 & Balluta & $35^{\circ} 54^{\prime} 56^{\prime \prime} \mathrm{N} 14^{\circ} 29^{\prime} 49^{\prime \prime} \mathrm{E}$ & $<_{10 \mathrm{~m}^{-2}}$ & Concrete wall \\
\hline 21.IX.2010 & Sliema Seafront, Sliema & $35^{\circ} 54^{\prime} 55^{\prime \prime} \mathrm{N} 14^{\circ} 30^{\prime} 24^{\prime \prime} \mathrm{E}$ & $\sim_{50 \mathrm{~m}^{-2}}$ & $\begin{array}{l}\text { Lower Globigerina Limestone } \\
\text { coastline }\end{array}$ \\
\hline 26.III.2011 & Qui-si-sana, Sliema & $35^{\circ} 54^{\prime} 41^{\prime \prime} \mathrm{N} 14^{\circ} 30^{\prime} 29^{\prime \prime} \mathrm{E}$ & $\sim 10 \mathrm{~m}^{-2}$ & $\begin{array}{l}\text { Lower Globigerina Limestone } \\
\text { coastline }\end{array}$ \\
\hline 26.III.2011 & & $35^{\circ} 54^{\prime} 41^{\prime \prime} \mathrm{N} 14^{\circ} 30^{\prime} 26^{\prime \prime} \mathrm{E}$ & $\sim_{10} \mathrm{~m}^{-2}$ & \\
\hline 26.III.2011 & & $35^{\circ} 54^{\prime} 40^{\prime \prime} \mathrm{N} 14^{\circ} 30^{\prime} 35^{\prime \prime} \mathrm{E}$ & $\sim_{100 \mathrm{~m}^{-2}}$ & \\
\hline 26.III.2011 & & $35^{\circ} 54^{\prime} 40^{\prime \prime} \mathrm{N} 14^{\circ} 30^{\prime} 32^{\prime \prime} \mathrm{E}$ & $\sim 10 \mathrm{~m}^{-2}$ & \\
\hline 26.III.2011 & & $35^{\circ} 54^{\prime} 37^{\prime \prime} \mathrm{N} 14^{\circ} 30^{\prime} 38^{\prime \prime} \mathrm{E}$ & $\sim 100 \mathrm{~m}^{-2}$ & \\
\hline 26.III.2011 & & $35^{\circ} 54^{\prime} 34^{\prime \prime} \mathrm{N} 14^{\circ} 30^{\prime} 40^{\prime \prime} \mathrm{E}$ & $\sim_{100 \mathrm{~m}^{-2}}$ & \\
\hline 26.III.2011 & Tignè Point, Sliema & $35^{\circ} 54^{\prime} 32^{\prime \prime} \mathrm{N} 14^{\circ} 30^{\prime} 41^{\prime \prime} \mathrm{E}$ & $\sim 100 \mathrm{~m}^{-2}$ & $\begin{array}{l}\text { Lower Globigerina Limestone } \\
\text { coastline }\end{array}$ \\
\hline 26.III.2011 & & $35^{\circ} 54^{\prime} 31^{\prime \prime} \mathrm{N} 14^{\circ} 30^{\prime} 43^{\prime \prime} \mathrm{E}$ & $\sim 10 \mathrm{~m}^{-2}$ & \\
\hline 26.III.2011 & $35^{\circ} 54^{\prime} 22^{\prime \prime} \mathrm{N} 14^{\circ} 30^{\prime} 34^{\prime \prime} \mathrm{E}$ & Not observed & Concrete wall & \\
\hline 26.III.2011 & & $35^{\circ} 54^{\prime} 21^{\prime \prime} \mathrm{N} 14^{\circ} 30^{\prime} 38^{\prime \prime} \mathrm{E}$ & $\sim_{10} \mathrm{~m}^{-2}$ & \\
\hline 26.III.2011 & & $35^{\circ} 54^{\prime} 20^{\prime \prime} \mathrm{N} 14^{\circ} 30^{\prime} 40^{\prime \prime} \mathrm{E}$ & None & $\begin{array}{l}\text { Lower Globigerina Limestone } \\
\text { coastline }\end{array}$ \\
\hline 26.III.2011 & $\begin{array}{l}35^{\circ} 54^{\prime} 19^{\prime \prime} \mathrm{N} 14^{\circ} 30^{\prime} 42^{\prime \prime} \mathrm{E} \text { to } \\
35^{\circ} 54^{\prime} 30^{\prime \prime} \mathrm{N} \\
14^{\circ} 30^{\prime} 43^{\prime \prime} \mathrm{E}\end{array}$ & Not observed & $\begin{array}{l}\text { Mostly Lower Globigerina } \\
\text { Limestone coastline } \\
\text { with concrete areas }\end{array}$ & \\
\hline 26.III.2011 & & $35^{\circ} 54^{\prime} 30^{\prime \prime} \mathrm{N} 14^{\circ} 30^{\prime} 43^{\prime \prime} \mathrm{E}$ & $\sim_{10 \mathrm{~m}^{-2}}$ & $\begin{array}{l}\text { Lower Globigerina Limestone } \\
\text { coastline }\end{array}$ \\
\hline 23.XII.2011 & Sliema Creek area & $35^{\circ} 54^{\prime} 31^{\prime \prime} \mathrm{N} 14^{\circ} 30^{\prime} 13^{\prime \prime} \mathrm{E}$ & None & Concrete steps \\
\hline 23.XII.2011 & & $35^{\circ} 54^{\prime} 27^{\prime \prime} \mathrm{N} 14^{\circ} 29^{\prime} 58^{\prime \prime} \mathrm{E}$ & Not observed & Concrete wall \\
\hline 23.XII.2011 & $35^{\circ} 54^{\prime} 15^{\prime \prime} \mathrm{N} 14^{\circ} 29^{\prime} 45^{\prime \prime} \mathrm{E}$ & None & Concrete and boulders & \\
\hline 23.XII.2011 & & $35^{\circ} 54^{\prime} 10^{\prime \prime} \mathrm{N} 14^{\circ} 29^{\prime} 44^{\prime \prime} \mathrm{E}$ & None & \\
\hline 23.XII.2011 & Ta' Xbiex & $35^{\circ} 54^{\prime} \mathrm{O}^{\prime \prime} \mathrm{N} 14^{\circ} 29^{\prime} 52^{\prime \prime} \mathrm{E}$ & None & Concrete wall \\
\hline
\end{tabular}


Table 2. Continued

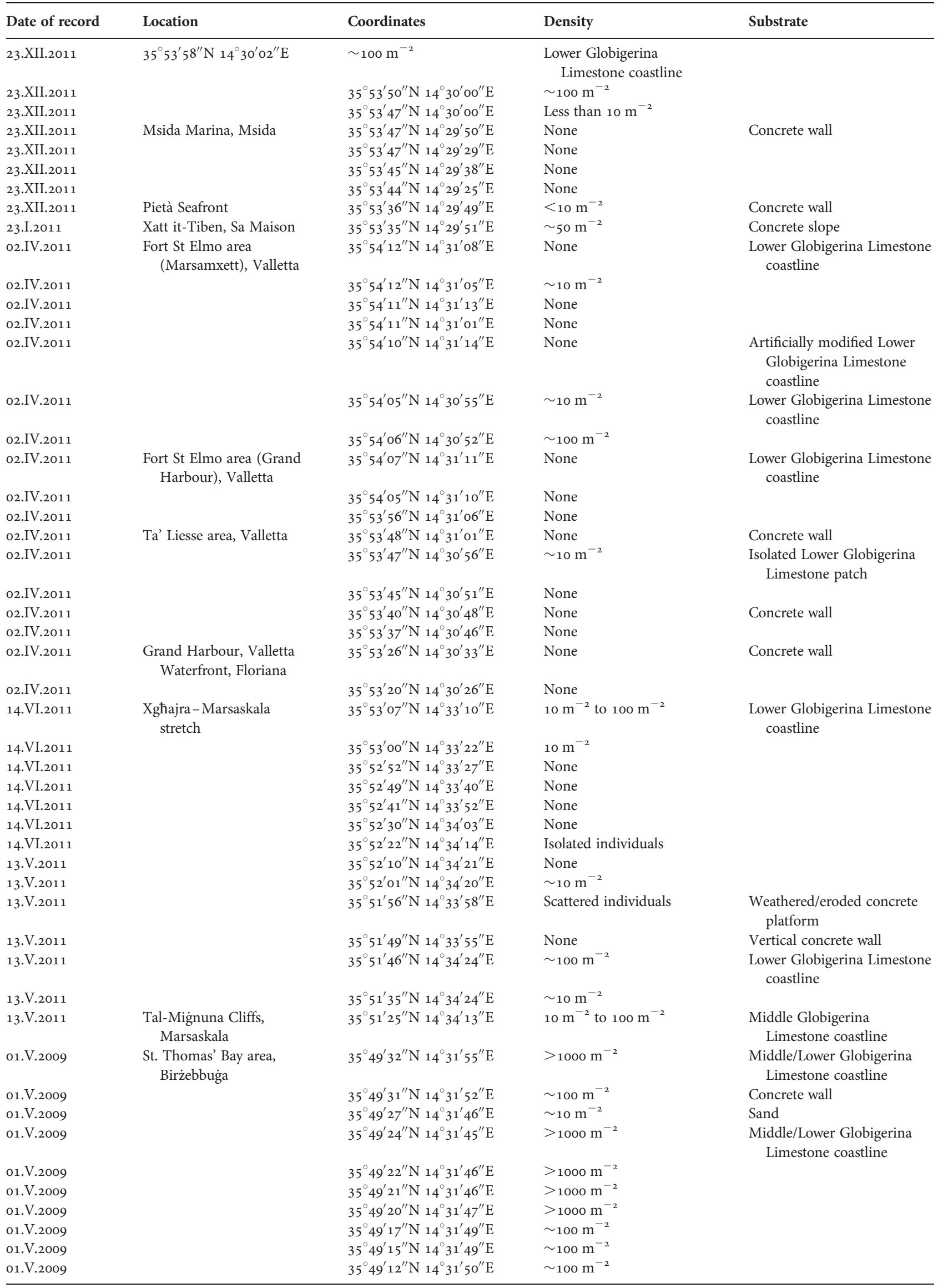


Table 2. Continued

\begin{tabular}{|c|c|c|c|c|}
\hline Date of record & Location & Coordinates & Density & Substrate \\
\hline 13.V.2011 & & $35^{\circ} 51^{\prime} 17^{\prime \prime} \mathrm{N} 14^{\circ} 34^{\prime} \mathrm{oo}^{\prime \prime} \mathrm{E}$ & $\sim 10 \mathrm{~m}^{-2}$ & \\
\hline 09.II.2011 & Xrobb 1-Ghagin, Delimara & $35^{\circ} 50^{\prime} 26^{\prime \prime} \mathrm{N} 14^{\circ} 34^{\prime} 07^{\prime \prime} \mathrm{E}$ & Scattered individuals & $\begin{array}{l}\text { Upper Globigerina Limestone, } \\
\text { with occasional Middle and } \\
\text { Lower Globigerina } \\
\text { Limestone outcrops }\end{array}$ \\
\hline 09.II.2011 & & $35^{\circ} 49^{\prime} 52^{\prime \prime} \mathrm{N} 14^{\circ} 33^{\prime} 33^{\prime \prime} \mathrm{E}$ & Scattered individuals & \\
\hline 09.II.2011 & $\begin{array}{c}\text { San Luċjan promontory, } \\
\text { Qajjenza, Birżebbuga }\end{array}$ & $35^{\circ} 50^{\prime} 18^{\prime \prime} \mathrm{N} 14^{\circ} 32^{\prime} 59^{\prime \prime} \mathrm{E}$ & None & $\begin{array}{l}\text { Middle Globigerina } \\
\text { Limestone large cobbles/ } \\
\text { stones/small boulders }\end{array}$ \\
\hline 09.II.2011 & & $35^{\circ} 50^{\prime} 10^{\prime \prime} \mathrm{N} 14^{\circ} 32^{\prime} 43^{\prime \prime} \mathrm{E}$ & Scattered individuals & $\begin{array}{l}\text { Concrete wall (relatively } \\
\text { smooth) }\end{array}$ \\
\hline 09.II.2011 & & $35^{\circ} 50^{\prime} \mathrm{O}^{\prime \prime} \mathrm{N} 14^{\circ} 33^{\prime} \mathrm{O} 4^{\prime \prime} \mathrm{E}$ & None & $\begin{array}{l}\text { Concrete wharf/jetty-in } \\
\text { ruins }\end{array}$ \\
\hline 09.II.2011 & & $35^{\circ} 49^{\prime} 59^{\prime \prime} \mathrm{N} 14^{\circ} 32^{\prime} 44^{\prime \prime} \mathrm{E}$ & Scattered individuals & $\begin{array}{l}\text { Upper Globigerina Limestone, } \\
\text { with occasional Middle and } \\
\text { Lower Globigerina } \\
\text { Limestone outcrops }\end{array}$ \\
\hline 04.II.2011 & & $35^{\circ} 49^{\prime} 55^{\prime \prime} \mathrm{N} 14^{\circ} 32^{\prime} 21^{\prime \prime} \mathrm{E}$ & $>_{1000 \mathrm{~m}^{-2}}$ & \\
\hline 04.II.2011 & & $35^{\circ} 49^{\prime} 46^{\prime \prime} \mathrm{N} 14^{\circ} 32^{\prime} 41^{\prime \prime} \mathrm{E}$ & $<10 \mathrm{~m}^{-2}$ & \\
\hline 09.II.2011 & & $35^{\circ} 49^{\prime} 53^{\prime \prime} \mathrm{N} 14^{\circ} 32^{\prime} 46^{\prime \prime} \mathrm{E}$ & $\sim 100 \mathrm{~m}^{-2}$ to $1000 \mathrm{~m}^{-2}$ & $\begin{array}{l}\text { Middle and Upper } \\
\text { Globigerina Limestone } \\
\text { platform }\end{array}$ \\
\hline $13 . V .2011$ & Munxar Point, Birżebbuga & $35^{\circ} 51^{\prime} \mathrm{oo}^{\prime \prime} \mathrm{N} 14^{\circ} 34^{\prime} 13^{\prime \prime} \mathrm{E}$ & $\sim 100 \mathrm{~m}^{-2}$ & $\begin{array}{l}\text { Middle Globigerina } \\
\text { Limestone coastline }\end{array}$ \\
\hline $13 . V .2011$ & & $35^{\circ} 51^{\prime} \mathrm{oo}^{\prime \prime} \mathrm{N} 14^{\circ} 34^{\prime} 13^{\prime \prime} \mathrm{E}$ & $\sim 100 \mathrm{~m}^{-2}$ & $\begin{array}{l}\text { Middle/Lower Globigerina } \\
\text { Limestone coastline }\end{array}$ \\
\hline $13 . V .2011$ & & $35^{\circ} 51^{\prime} \mathrm{Oo}^{\prime \prime} \mathrm{N} 14^{\circ} 34^{\prime} \mathrm{O} 2^{\prime \prime} \mathrm{E}$ & $\sim_{10} \mathrm{~m}^{-2}$ & $\begin{array}{l}\text { Middle Globigerina } \\
\text { Limestone coastline }\end{array}$ \\
\hline 13.V.2011 & & $35^{\circ} 51^{\prime} \mathrm{Oo}^{\prime \prime} \mathrm{N} 14^{\circ} 34^{\prime} \mathrm{O}^{\prime \prime} \mathrm{E}$ & $\sim_{10} \mathrm{~m}^{-2}$ & $\begin{array}{l}\text { Middle/Lower Globigerina } \\
\text { Limestone coastline }\end{array}$ \\
\hline 09.II.2011 & & $35^{\circ} 50^{\prime} 14^{\prime \prime} \mathrm{N} 14^{\circ} 33^{\prime} 48^{\prime \prime} \mathrm{E}$ & Scattered individuals & \\
\hline 09.II.2011 & $\begin{array}{l}\text { St George's Bay, Qajjenza, } \\
\text { Birżebbugia }\end{array}$ & $35^{\circ} 49^{\prime} 58^{\prime \prime} \mathrm{N} 14^{\circ} 32^{\prime} 13^{\prime \prime} \mathrm{E}$ & $\sim 10 \mathrm{~m}^{-2}$ & $\begin{array}{l}\text { Upper Globigerina Limestone, } \\
\text { with occasional Middle and } \\
\text { Lower Globigerina } \\
\text { Limestone outcrops }\end{array}$ \\
\hline 09.II.2011 & & $35^{\circ} 49^{\prime} 57^{\prime \prime} \mathrm{N} 14^{\circ} 32^{\prime} 17^{\prime \prime} \mathrm{E}$ & $>_{1000 \mathrm{~m}^{-2}}$ & \\
\hline 09.II.2011 & & $35^{\circ} 49^{\prime} 56^{\prime \prime} \mathrm{N} 14^{\circ} 32^{\prime} \mathrm{o} 6^{\prime \prime} \mathrm{E}$ & $>_{1000 \mathrm{~m}^{-2}}$ & \\
\hline 09.II.2011 & & $35^{\circ} 49^{\prime} 56^{\prime \prime} \mathrm{N} 14^{\circ} 32^{\prime} \mathrm{oo}^{\prime \prime} \mathrm{E}$ & $\sim 100 \mathrm{~m}^{-2}$ & \\
\hline 09.II.2011 & & $35^{\circ} 49^{\prime} 56^{\prime \prime} \mathrm{N} 14^{\circ} 31^{\prime} 57^{\prime \prime} \mathrm{E}$ & $\sim 10 \mathrm{~m}^{-2}$ & \\
\hline 29.VI.2011 & Maghlaq promontories & $35^{\circ} 49^{\prime} 34^{\prime \prime} \mathrm{N} 14^{\circ} 25^{\prime} 51^{\prime \prime} \mathrm{E}$ & None & $\begin{array}{l}\text { Lower Coralline Limestone } \\
\text { coastline }\end{array}$ \\
\hline 29.VI.2011 & & $35^{\circ} 49^{\prime} 33^{\prime \prime} \mathrm{N} 14^{\circ} 25^{\prime} 53^{\prime \prime} \mathrm{E}$ & None & \\
\hline 29.VI.2011 & & $35^{\circ} 49^{\prime} 32^{\prime \prime} \mathrm{N} 14^{\circ} 25^{\prime} 55^{\prime \prime} \mathrm{E}$ & None & \\
\hline $13 . V .2011$ & Ghar Lapsi main area & $35^{\circ} 49^{\prime} 38^{\prime \prime} \mathrm{N} 14^{\circ} 25^{\prime} 25^{\prime \prime} \mathrm{E}$ & $\sim_{10 \mathrm{~m}^{-2}}$ & $\begin{array}{l}\text { Weathered/eroded concrete } \\
\text { platform }\end{array}$ \\
\hline 29.I.2011 & $\begin{array}{l}\text { Fomm ir-Rih-Anchor } \\
\text { Bay stretch }\end{array}$ & $\begin{array}{l}35^{\circ} 54^{\prime} 48^{\prime \prime} \mathrm{N} 14^{\circ} 20^{\prime} 15^{\prime \prime} \mathrm{E} \\
\text { to } 35^{\circ} 57^{\prime} 35^{\prime \prime} \mathrm{N} \\
14^{\circ} 20^{\prime} 20^{\prime \prime} \mathrm{E}\end{array}$ & None & $\begin{array}{l}\text { Isolated Upper, Middle and } \\
\text { Lower Globigerina } \\
\text { Limestone outcrops along } \\
\text { extensive coastal stretch }\end{array}$ \\
\hline 05.III.2011 & $\begin{array}{l}\text { Ghajn Tuffieha to } \\
\text { Il-Mixquqa area, } \\
\text { Mellieћa }\end{array}$ & $35^{\circ} 55^{\prime} 47^{\prime \prime} \mathrm{N} 14^{\circ} 20^{\prime} 40^{\prime \prime} \mathrm{E}$ & None & $\begin{array}{l}\text { Upper Coralline Limestone } \\
\text { boulders and pebbles }\end{array}$ \\
\hline 05.III.2011 & & $35^{\circ} 55^{\prime} 43^{\prime \prime} \mathrm{N} 14^{\circ} 20^{\prime} 40^{\prime \prime} \mathrm{E}$ & None & Sand \\
\hline 05.III.2011 & & $35^{\circ} 55^{\prime} 40^{\prime \prime} \mathrm{N} 14^{\circ} 20^{\prime} 34^{\prime \prime} \mathrm{E}$ & $\sim 10 \mathrm{~m}^{-2}$ & $\begin{array}{l}\text { Upper Coralline Limestone } \\
\text { boulders and pebbles }\end{array}$ \\
\hline 05.III.2011 & & $35^{\circ} 55^{\prime} 34^{\prime \prime} \mathrm{N} 14^{\circ} 20^{\prime} 27^{\prime \prime} \mathrm{E}$ & None & \\
\hline
\end{tabular}

The physical conditions in the sheltered area near Manoel Island, which is lacking in $B$. pharaonis, are similar to others where large densities of the species may be found. The bivalve's absence may be due to the extensive populations of Anemonia viridis Forsskål, 1775 which may preclude the larvae from settling.
A gastropod which may actively inhibit the settlement of $B$. pharaonis larvae is the predatory Pisania striata (Gmelin, 1791), whose presence in the current study mostly coincided with the absence or a relative scarcity of the mytilid. On the other hand, Osilinus turbinatus (Born, 1778), Muricopsis cristata (Brocchi, 1814), Cerithium lividulum Risso, 1826, 
patellids, chitons, and Chthamalus spp. were very frequently observed inhabiting the same areas as B. pharaonis. Bonnici (unpublished data) reports the following molluscan species as being exclusively reported from $B$. pharaonis beds (i.e. these species were missing from reference locations where $B$. pharaonis was only present as sparse individuals and not as beds): O. turbinatus, Ocinebrina edwardsii (Payraudeau, 1826) and Patella spp., together with Podocerus sp., nereids, nemerteans and sabellids.

A variety of macrofloral species characterized shores with $B$. pharaonis, with the main dominant species being Corallina elongata Ellis \& Solander, Cladophora prolifera (Roth) Kützing, Ceramium ciliatum (Ellis) Ducluzeau, Ulva/Enteromorpha spp. and Jania rubens (Linné) Lamouroux along sheltered and polluted shores, and Cystoseira spp., Padina pavonica (Linné) Thivy, Sargassum vulgare C. Agardh and Dictyopteris polypodioides (De Candolle) Lamouroux along more exposed shores.

Besides ecological interactions, tolerance to the prevailing physical conditions obviously shape the current distribution of $B$. pharaonis, with exposure to wave action and phytoplankton quantity seemingly being the most important distribution determinants for the species. In fact, according to CIESM (2003) 'its abundance seems to be negatively associated with wave exposure'. The main repositories of $B$. pharaonis individuals recorded in the current study were sheltered areas, mainly within large embayments (Marsaxlokk Bay, Grand Harbour, Marsamxett Harbour and Salina), characterized by significant levels of water pollution in view of their popularity for shipping and recreational vessel berthing. Large breaks in the distribution of $B$. pharaonis have been recorded along the most exposed shores of the island of Malta (e.g. Pembroke-refer to Table 2). This finding is consistent with that of Safriel \& Sasson-Frostig (1988) but contradicts that reported by Rilov et al. (2004), who report extensive beds of $B$. pharaonis from wave-exposed stretches of the Israeli coastline. Bonnici (unpublished data) reports no significant variation between wave exposure values for locations with mussel beds formations and with sparse individuals.

\section{ACKNOWLEDGEMENTS}

The authors are grateful to the following individuals for assistance in work related to this study: $\mathrm{Mr}$ Edwin Lanfranco (identification of algal specimens), Mr Arnold Sciberras and Mr Jeffrey Sciberras (sampling duties), and Mr Charles Cachia and Mr Constantine Mifsud (provision of local records of the species).

\section{REFERENCES}

Cachia C. (1975) Further notes on Maltese Mollusca. The Maltese Naturalist 2, 28-30.

Cachia C. (1999) Il-Molluski ta' Malta. Kullana Kulturali, 5. Malta: Pubblikazzjonijiet Indipendenza, $\mathrm{x}+210 \mathrm{pp}$.

Cachia C., Mifsud C. and Sammut P.M. (1993) An annotated checklist of the marine Mollusca of the Maltese Islands. Rankweil, Austria: Erste Vorarlberger Malakologische Gesellschaft, pp. 1-180.

Cachia C., Mifsud C. and Sammut P.M. (2004) The marine Mollusca of the Maltese Islands Part 4: Caudofoveata, Solenogastres, Bivalvia,
Scaphopoda and Cephalopoda. Leiden, The Netherlands: Backhuys Publishers, pp. 1-270.

CIESM (2003) CIESM Atlas of exotic species in the Mediterranean. Volume 3. Molluscs. (Edited by Zenatos A., Gafas S., Russo G. and Templado J. for CIESM) Monaco: CIESM Publishers, pp. 1-376.

De Min R. and Vio E. (1997) Molluschi conchiferi del litorale sloveno. Annals for Istran and Mediterranean Studies 11, 241-258.

Dogan A., Onen M. and Ozturk B. (2007) A new record of the invasive Red Sea mussel Brachidontes pharaonis (Fischer P., 1870) (Bivalvia: Mytilidae) from the Turkish coasts. Aquatic Invasions 2, 461-463.

Felsenburg T. and Safriel U. (1974) Colonization of eastern Mediterranean intertidal zone by Indo-Pacific mussel, Brachidontes variabilis. Israel Journal of Zoology 23, 212-213.

Gilboa A. (1976) Experiments in mytilids' recolonization. MSc thesis. Hebrew University of Jerusalem.

Google Maps (2011) Map of Malta. Available at: http://maps.google.com/ (accessed 15 December 2011)

Government of Malta (1993) Geological map of the Maltese Islands. 2 sheets, 1:2500o. Malta: Oil Exploration Directorate, Office of the Prime Minister.

Huber M. (2010) Compendium of bivalves-a full-color guide to 3,300 of the world's marine bivalves-a status on Bivalvia after 250 years of research. Hackenheim, Germany: ConchBooks, 901 pp. + CD.

Krauss F. (1848) Die Südafrikanischen Mollusken.Ein Beitrag zur Kenntniss der Mollusken des Kap- und Natallandes und zur Geographischen Verbreitung Derselben mit Beschreibung und Abbildung der Neuen Arten. Stuttgart: Ebner and Seubert, 140 pp. +6 pls.

Lamy E. (1919) Les Moules et les Modioles de la Mer Rouge (d'après les Matériaux recuellis par M. le Dr. Jousseaume). Bulletin du Muséum national d'Histoire naturelle, Paris 25, 173-178.

Lamy E. (1936) Révision des Mytilidae vivants du Muséum National d'Histoire Naturelle de Paris. Journal de Conchyliologie 80, 66-102; $107-198 ; 229-295$.

Lanfranco G. (1975) Some additions to the local Mollusca. The Maltese Naturalist 2, 27.

Mifsud C. and Cilia D.P. (2009) On the presence of a colony of Brachidontes pharaonis (P. Fischer, 1870) (Bivalvia: Mytilidae) in Maltese waters (Central Mediterranean). Triton 20, 20-22.

Morton B. (1988) The population dynamics and reproductive cycle of Brachidontes variabilis (Bivalvia: Mytilidae) in a Hong Kong Mangrove. Malacological Review 21, 109-117.

Por F.D. (1978) Lessepsian migration. The influx of Red Sea biota into the Mediterranean by way of the Suez Canal. Ecological Studies 23, 1-228.

Rilov G., Gasith A. and Benayahu Y. (2002) Effect of an exotic prey on the feeding pattern of a predatory snail. Marine Environmental Research 54, 85-98.

Rilov G., Benayahu Y. and Gasith A. (2004) Prolonged lag in population outbreak of an invasive mussel: a shifting habitat model. Biological Invasions 6, 347-364.

Safriel U.N., Gilboa F. and Felsenburg D. (1980) Distribution of rocky intertidal mussels in the Red Sea coasts of Sinai, the Suez Canal and the Mediterranean coast of Israel, with special reference to recent colonizers. Journal of Biogeography 7, 39-62.

Safriel U.N. and Sasson-Frostig Z. (1988) Can colonizing mussel outcompete indigenous mussel? Journal of Experimental Marine Biology and Ecology 117, 211-226. 
Sarà G. (2006) Hydrodynamic effect on the origin and quality of organic matter for bivalves: an integrated isotopic, biochemical and transplant study. Marine Ecology Progress Series 328, 65-73.

Sarà G., Romano C., Caruso M. and Mazzola A. (2000) The new Lessepsian entry Brachidontes pharaonis (Fischer P., 1870) (Bivalvia, Mytilidae) in the western Mediterranean: a physiological analysis under varying natural conditions. Journal of Shellfish Research 19, 967-977.

Sarà G., Romano C. and Mazzola A. (2008) A new Lessepsian species in the western Mediterranean (Brachidontes pharaonis Bivalvia, Mytilidae): density, resource allocation and biomass. JMBA Marine Biodiversity Records 1, e8. DOI: 10.1017/S175526720600087X.

Sarà G., Vizzini S. and Mazzola A. (2003) Sources of carbon and dietary habits of new Lessepsian entry Brachidontes pharaonis (Bivalvia, Mytilidae) in the western Mediterranean. Marine Biology 143, $713-722$.

Shefer S. (2003) Factors and processes facilitate the invasion of Brachidontes pharaonis into the Levant basin. PhD thesis. Tel Aviv University, Israel.

Shefer S., Abelson A., Mokady O. and Geffen E. (2004) Red to Mediterranean Sea bioinvasion: natural drift through the Suez Canal, or anthropogenic transport? Molecular Ecology 13, 2333-2343.

Sciberras M. and Schembri P.J. (2007) A critical review of records of alien marine species from the Maltese Islands and surrounding waters (Central Mediterranean). Mediterranean Marine Science 8, 41-66.
Sirna Terranova M., Lo Brutto S., Arculeo M. and Mitton J.B. (2006) Population structure of Brachidontes pharaonis (P. Fischer, 1870) (Bivalvia, Mytilidae) in the Mediterranean Sea, and evolution of a novel mtDNA polymorphism. Marine Biology 159, 89-101.

Sirna Terranova M., Lo Brutto S., Arculeo M. and Mitton J.B. (2007) A mitochondrial phylogeography of Brachidontes variabilis (Bivalvia: Mytilidae) reveals three cryptic species. Journal of Zoological Systematics and Evolutionary Research 45, 289-298.

Tenekides N.S. (1989) On a collection of shells from the Greek seas. Athens: Protopapa Press, $187 \mathrm{pp}$.

and

Zenetos A., Gofas S., Verlaque M., Cinar M. E., Garcia Raso E., Bianchi C.N., Morri C., Azzurro E., Bilecenoglu M., Froglia C., Siokou I., Violanti D., Sfriso A., San Martin A., Giangrande A., Katagan T., Ballesteros E., Ramos Espla A., Mastrototaro F., Ocana O., Zingone A., Gambi M.C. and Streftaris N. (2010) Alien species in the Mediterranean Sea by 2010. A contribution to the application of European Union's Marine Strategy Framework Directive (MSFD). Part I. Spatial distribution. Mediterranean Marine Science 11, 381-493.

\section{Correspondence should be addressed to:}

A. Deidun

International Ocean Institute-Malta Operational Centre University of Malta, Msida, MSD 2080, Malta email: alandeidun@gmail.com 